\title{
Archéopages
}

Archéopages

Archéologie et société

40 | 04-07/2014

Villages

\section{Villages du Néolithique en Méditerranée occidentale. Sédentarisation et habitat groupé}

Jean Vaquer et Muriel Gandelin

\section{(2) OpenEdition}

1 Journals

Édition électronique

URL : https://journals.openedition.org/archeopages/585

DOI : 10.4000/archeopages.585

ISSN : 2269-9872

Éditeur

INRAP - Institut national de recherches archéologiques préventives

Édition imprimée

Date de publication : 1 juin 2015

Pagination : 6-15

ISSN : 1622-8545

\section{Référence électronique}

Jean Vaquer et Muriel Gandelin, « Villages du Néolithique en Méditerranée occidentale.

Sédentarisation et habitat groupé ", Archéopages [En ligne], 40 | 04-07/2014, mis en ligne le 01 juillet

2016, consulté le 21 janvier 2022. URL : http://journals.openedition.org/archeopages/585; DOI :

https://doi.org/10.4000/archeopages.585 


\title{
Villages du Néolithique \\ en Méditerranée occidentale Sédentarisation et habitat groupé
}

\author{
Jean Vaquer CNRS, UMR 5608 «Travaux et recherches archéologiques sur les cultures, les sspacese et les sociétés»" \\ Muriel Gandelin CNRS, UMR 5608 «Travaux et recherches archéologiques sur les cultures, les espaces et les sociétés»
}

Si le mot village dans sa signification actuelle et occidentale correspond à un groupe d'habitats abritant une communauté rassemblée par des intérêts communs et sous une autorité politique, en milieu rural, il se définit aussi comme une forme intermédiaire entre les fermes ou les «masages» et hameaux qui sont dépourvus d'organe de direction autres que familiaux et des formes plus importantes d'habitat groupé comme les bourgs ou les petites villes ayant des services publics plus diversifiés, notamment un marché et des structures d'administration plus complexes. Dans ces conditions, l'emploi du mot «village» pour les périodes préhistoriques est évidemment anachronique et pour le moins inadapté puisque plusieurs critères sociologiques ou politiques qui le définissent ne sont pas accessibles par les méthodes archéologiques. Si on veut l'utiliser, ce ne peut être que dans une acception générale d'habitat groupé plus ou moins stabilisé et pour le distinguer des habitats plus petits ou isolés, probablement peu durables, qui étaient sans doute mieux adaptés à une agriculture et un élevage sur abattis-brûlis. Ces «villages» seraient donc les pôles fixes d'une société dont les techniques imposaient pour la plupart une mobilité ou une itinérance des installations. Faut-il alors préférer d'autres termes pour qualifier les habitats groupés du Néolithique et tenter de cerner des formes types de ces habitats groupés par rapport à la multitude des habitats dispersés?

Plusieurs termes sont d'usage courant dans la discipline mais se référent à d'autres réalités qui tiennent plus à leur aspect archéologique qu'à leur position fonctionnelle dans la structuration ancienne de l'habitat. C'est ainsi que l'on utilise fréquemment le mot «tell» pour des habitats groupés en terre crue qui se sont superposés en finissant par former des buttes artificielles, le terme "palafitte» pour des habitats groupés en bois établis dans les milieux humides, les «enceintes à fossés » pour les systèmes fortifiés le plus souvent arasés dont il ne subsiste que les structures fossoyées, les «camps de hauteur » et les «éperons barrés » pour les systèmes fortifiés en position dominante comportant des défenses en relief, les «villages en pierre sèche " pour les habitats chalcolithiques des zones calcaires du Languedoc. Ces dénominations ont du sens et correspondent à des réalités archéologiques qui se retrouvent à de multiples exemplaires et pour lesquels des façons particulières de fouiller et de tirer parti des faits archéologiques ont été mises au point. Elles ne peuvent cependant pas prétendre qualifier toute la diversité et la complexité des formes d'habitat qui ont pu exister, ni permettre de rendre compte du mode d'occupation des territoires et de la structuration réelle de l'habitat à des échelles qui dépassent les microzones. Il est évident, par ailleurs, que la très grande majorité des sites archéologiques n'entre pas dans ces diverses catégories emblématiques en raison de la seule présence de fosses, de l'impossibilité de reconnaître des maisons, de l'absence de limite nette, d'un nombre réduit de structures et des difficultés à établir leur chronologie et donc $a$ fortiori leur synchronie.

\section{L'habitat, reflet des organisations sociales et de l'économie \\ Il est couramment admis pourtant que les} groupes humains du Néolithique étaient organisés en communautés villageoises. L'habitat groupé et plus ou moins fixé aurait même été un des facteurs essentiels de l'invention de l'économie néolithique fondée sur la domestication, puis sur le 
développement conjoint de l'agriculture et de l'élevage. C'est aussi ce mode d'organisation villageoise qui aurait été la cellule de base dans le processus d'expansion du Néolithique selon le principe de la «vague d'expansion démique» d'Ammerman et Cavalli-Sforza (1984). Dans le cadre d'une économie ayant un mode de production domestique foncièrement autarcique et un système agropastoral basé sur l'abattis-brûlis, c'est l'impossibilité de dépasser le niveau d'organisation villageoise qui aurait été la cause de la segmentation des communautés au-delà d'un seuil de quelques centaines de personnes et de l'essaimage de nouvelles communautés dans les territoires vierges disponibles tout au long du développement du Néolithique.

En considérant que le «village» au sens d'habitat groupé et structuré sous une autorité de niveau communautaire a été le noyau dur de la société néolithique, la principale question à se poser est de savoir si sa conception correspond à un ou plusieurs modèles et si ces derniers ont été diffusés en même temps que l'expansion du Néolithique. Pour aborder cette question, nous considérerons ici trois des principales formes d'habitats groupés ayant des limites clairement matérialisées. Ce qui permet, de ce fait, d'être assuré d'une organisation collective préconçue sur un espace suffisant pour une communauté villageoise.

\section{Les villages retranchés par des fossés et des murs}

En Italie du Sud, notamment dans La Daunia (région du nord des Pouilles autour de Foggia), un très grand nombre de sites néolithiques a été détecté grâce aux clichés aériens militaires pris par la Royal Air Force au cours de la Seconde Guerre mondiale. Ils ont permis d'élaborer une typologie des établissements bien visibles grâce à la conjonction de plusieurs facteurs favorables à la détection et au relevé complet des sites (Bradford,Williams-Hunt, 1946). Les fouilles qui ont été réalisées par la suite ont permis de retracer l'évolution de ces systèmes retranchés au cours des $\mathrm{VI}^{\mathrm{e}}$ et $\mathrm{V}^{\mathrm{e}}$ millénaires et de recueillir des informations sur l'articulation fonctionnelle de ces diverses structures. Au niveau le plus bas se trouvent des petits fossés ouverts dits en C qui, sous l'influence des archéologues anglais, ont été nommés «compound» au sens de concession familiale. Mesurant entre 15 et $40 \mathrm{~m}$ de diamètre, ils se composent d'un fossé qui devait être doublé par un mur sur son côté interne et qui limite un espace de quelques centaines de mètres carrés pouvant comporter les traces d'une unique habitation du type maison à abside et des structures annexes telles qu'un puits, des silos, un grenier, des aires pavées, des structures de combustion et de travail. Dans ces aires correspondant à l'enclos d'une maisonnée, le rôle du fossé n'est pas totalement éclairci: il semble qu'il n'était pas seulement une limite, mais aussi une structure fonctionnelle comme lieu d'extraction de matériau et de gestion de l'eau, ou pour fabriquer de la bauge ou du torchis [ill. 1].

D'autres enceintes à fossés creusés dans le sol meuble, mais aussi parfois dans la roche compacte, sont de plus grande ampleur et considérées comme des retranchements d'établissements villageois. Leur forme est circulaire à ovalaire et ils peuvent avoir plusieurs fossés concentriques, voire des enceintes annexes accolées. Ceux du début du vi ${ }^{\mathrm{e}}$ millénaire sont les plus simples et les plus petits, ils mesurent entre 80 et $120 \mathrm{~m}$ de diamètre. Dans celui de Rippa Tetta (Lucera), le fossé était doublé par un mur intérieur et l'aire interne comporte des structures diverses : maison en torchis, fours, aires empierrées. Vers la fin du vi millénaire, les villages retranchés deviennent beaucoup plus grands et ont une structure comportant plusieurs aires. Certaines enceintes enserrant des compounds groupés sont considérées comme les espaces villageois, tandis que les enceintes annexes vides de structures seraient des enclos à vocation économique. Le plus connu de ces grands sites ceinturés est Passo di Corvo (Foggia, Italie). Établi sur le rebord d'une terrasse, le site de Passo di Corvo est l'une des plus grandes enceintes fossoyées du Néolithique du Tavoliere. Un très long fossé externe de $6 \mathrm{~m}$ de large et de près de 5 $\mathrm{km}$ de long délimite une enceinte ovoïde vide de structures qui s'étend sur près 130 ha [ill. 2]. Au sudouest, un compartiment emboîté d'une quarantaine d'hectares, lui aussi ovoïde, est limité par trois fossés qui enserre une centaine de compounds. Cette partie est considérée comme le village d'une communauté d'environ 400 personnes, tandis que le grand enclos aurait servi pour des activités économiques comme des cultures particulières ou le gardiennage de troupeaux (Tiné, 1983). Les sondages et les fouilles ont montré que l'occupation de ce site avait débuté vers 5700 avant notre ère par les petites enceintes circulaires de Campo dei Fiori et de Podere Fredella. La principale occupation de Passo di Corvo se situe à la charnière des $\mathrm{VI}^{\mathrm{e}}$ et $\mathrm{V}^{\mathrm{e}}$ millénaires entre 5200 et 4800 avant notre ère. Cette longue durée se traduit notamment par des recreusements des fossés des compounds qui montrent la permanence de l'habitat au cours de plusieurs phases du début du Néolithique moyen.

En Italie méridionale, les enceintes à fossé sont encore attestées durant le Néolithique moyen au $\mathrm{V}^{\mathrm{e}}$ millénaire avant notre ère, mais elles deviennent moins fréquentes par rapport aux sites ouverts ou aux sites à habitats dispersés qui devaient être plus adaptés à une agriculture itinérante. Dans le Néolithique ancien du midi de la France, on connaît très peu d'habitats de plein air et aucun ne peut être classé dans la catégorie des grands sites ceinturés. Les sites de Portiragnes (Hérault), attribuables à l'étape des premières colonisations en provenance d'Italie péninsulaire, 
1. Exemple de compound fouillé sur le site de Passo di Corvo (Foggia, Pouilles, Italie). Ce type d'enclos familial se définit surtout par son fossé en C qui devait être bordé sur le côté interne et complété du côté ouvert par un mur en élévation dont on a retrouvé des vestiges. Dans ce cas, deux

compounds ont été

réalisés successivement

au même endroit et l'on a

pris soin de construire un

mur à l'endroit du

recoupement pour que le

fossé reste ouvert, ce qui

témoigne de son

importance pour drainer

et conserver l'eau. L'aire

interne contenait un puits

et à chaque compound

correspondait une petite

habitation allongée à

abside.

2. Passo di Corvo, Foggia, Pouilles (Italie). Cette vue aérienne du site prise par la Royal Air Force en 1943 a été traitée et recolorée pour mieux faire

apparaître les anomalies

de croissance des

céréales qui révèlent les

structures néolithiques.

Cela a permis de proposer

une nouvelle photo-

interprétation de ce

village qui s'étend sur

130 ha.
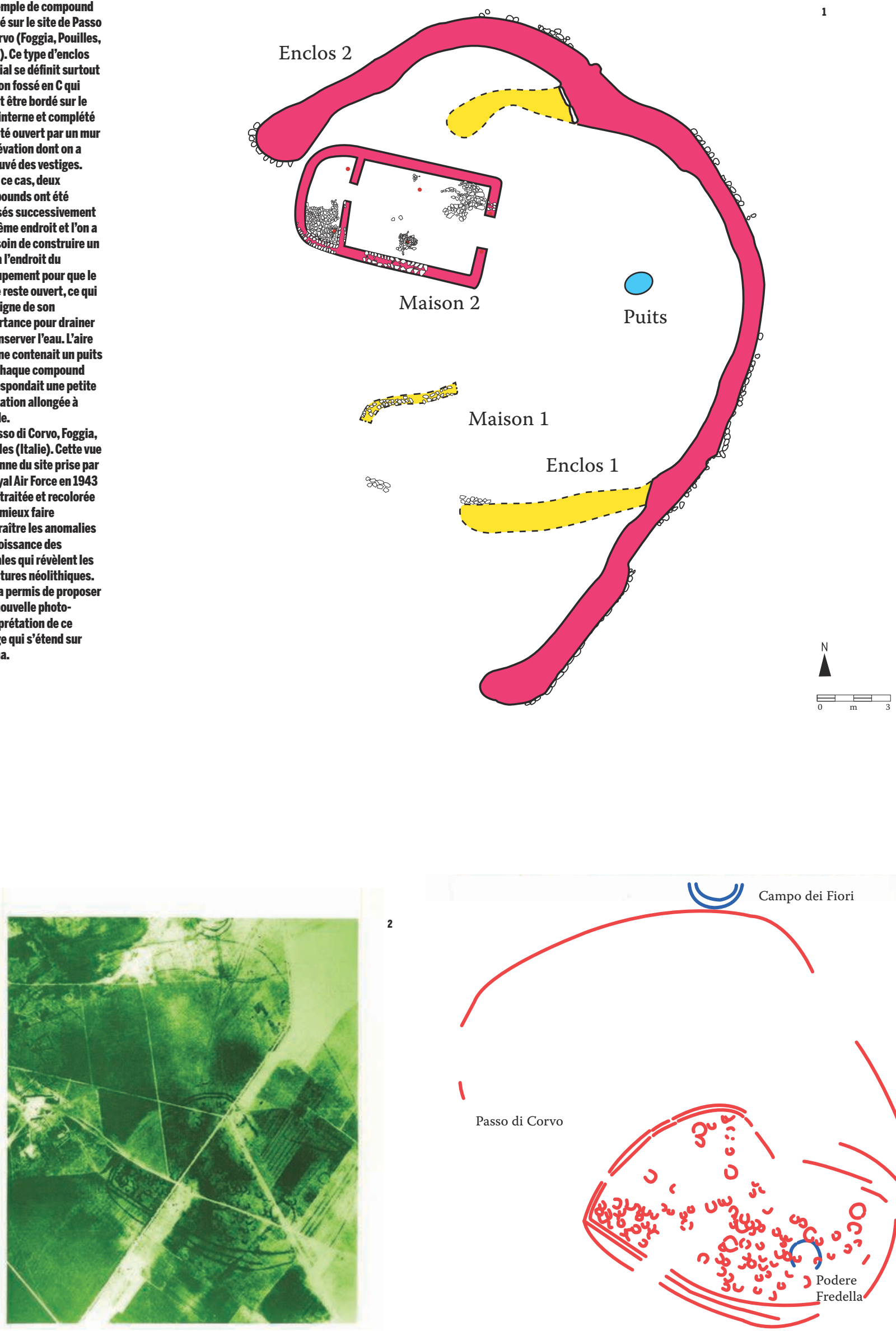

2

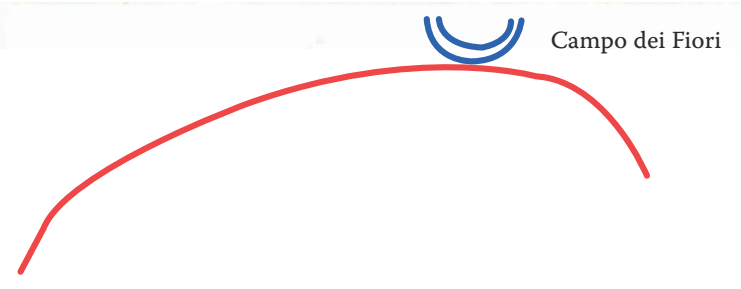

1

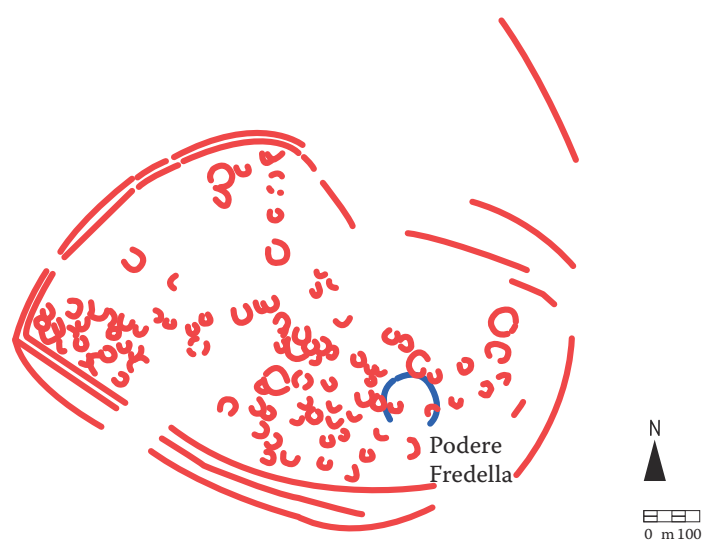


sont caractérisés par des petits regroupements de silos (Pont de Roque-Haute) ou par une grande fosse d'extraction d'argile (Peiro Signado) et sont d'extension trop modeste pour être autre chose que des habitats dispersés. Il en est de même pour les rares habitats de plein air du Cardial qui sont connus, hormis ceux de milieux humides. En Espagne, dans la région d'Alicante, le site du Mas d'Is à Penàguila (Alicante) est souvent cité comme une enceinte à fossé du Néolithique ancien. Ce site de près de 1 ha est une étroite bande de terrain marneux bordée par deux ravins qui a servi d'habitat aux vi et $\mathrm{v}^{\mathrm{e}}$ millénaires (Bernabeu Auban et al., 2003). Des restes de maisons à abside à ossature de poteaux et de foyers empierrés du Néolithique cardial ont été dégagés. Il y a aussi des tronçons de plusieurs fossés pouvant atteindre, pour les plus imposants, plus de $10 \mathrm{~m}$ de large et plus de $3 \mathrm{~m}$ de profondeur, mais ces derniers ne sont pas disposés en barrage d'éperon. Il a été envisagé qu'ils auraient pu avoir un développement circulaire en considérant qu'ils seraient mal conservés et que la topographie actuelle du site serait liée à l'érosion récente, ce qui paraît spéculatif. S’il est indéniable que ces fossés contiennent des rejets anthropiques des habitats qui se sont succédé sur le site, ils n'ont pas une forme très régulière et présentent des traces de ravinement et des couches de comblement échelonné sur une longue période, ce qui les rend différents des enceintes italiennes de cette époque. Au Portugal, depuis la découverte aérienne de la fameuse enceinte à fossés concentriques de Perdigões à Reguengos de Monserraz en 1996, les prospections et recherches sur photographies aériennes se sont multipliées et ont permis de détecter plus d'une cinquantaine de nouveaux sites à fossé. Si la plupart datent du Néolithique final et $\mathrm{du}$ Chalcolithique, d'autres reconnus partiellement au cours de sondages comme Senhora da Alegria (Coimbra) sont beaucoup plus anciens et datent du Néolithique ancien et du Néolithique moyen. Il s'agit toutefois de très petits fossés qui ne sont peut-être que des palissades (Valera, 2013) et leur lien avec des habitats de type villageois n'est pas établi. Ces sites n'ont pas de parenté avec ceux connus en Italie à la même période.

\section{Les villages ceinturés de palissade, en bois avec fossé et levée de terre}

En Italie du Nord, quelques tronçons de fossés ou de petits canaux de drainage sont attestés sur plusieurs sites du Néolithique ancien et moyen, mais ils sont bien différents des compounds et fossés d'enceinte du sud. Dans cette région, les systèmes de limitation des villages sont à matériaux mixtes, principalement le bois et la terre. Le site de Lugo di Romagna (Ravenna) est un bon exemple de site fortifié du Néolithique de cette région [ill. 3]. Il est un des habitats néolithiques les mieux conservés de l'Italie du Nord (Degaspari et $a l ., 1999)$. Occupé vers la fin du VI ${ }^{\mathrm{e}}$ millénaire, à l'époque de la culture de Fiorano, il comporte une enceinte de protection observée sur un tronçon de $40 \mathrm{~m}$ qui se composait d'un fossé creusé dans l'argile et qui a servi à l'édification d'une modeste levée de terre armée de petits rondins et de clayonnages. Ce dispositif a été complété par une palissade à poteaux jointifs faits de troncs refendus de chênes exceptionnellement conservés qui devait mesurer $3 \mathrm{~m}$ de hauteur hors sol et comportait une entrée en corridor. Une maison rectangulaire de 11 x $7 \mathrm{~m}$ se trouvait à l'intérieur du site. Elle était bâtie en torchis sur une armature de clayonnage et devait avoir un toit à double pente soutenu par les murs et quelques poteaux. Il s'agit là d'un modèle d'enceinte à palissade de bois, levée de terre et fossé qui est attesté aussi sur un autre site de la culture de Fiorano, celui de Lugo de Grezanna (Verona) et qui a été adopté par la suite dans d'autres contextes du Néolithique moyen occidental.

Dans le midi de la France, des enclos curvilignes comportant seulement une tranchée de palissade figurent parmi les structures les plus anciennes des grands habitats fortifiés du Néolithique moyen du Toulousain. Elles peuvent avoir une configuration en barrage d'éperon, comme à Saint-Michel-duTouch à Toulouse en Haute-Garonne [ill. 4], ou bien faire partie d'enclos circulaires comme le suggère la forme de celui reconnu partiellement à VilleneuveTolosane en Haute-Garonne (Gandelin, 2011). C'est au cours de la seconde moitié $d u v^{\mathrm{e}}$ millénaire que l'on voit apparaître des sites à enceinte segmentée. Celle de Poste Vieille à Pezens dans l'Aude, qui est attribuable au groupe de Bize, doit se situer vers 4000 avant notre ère (Guilaine, Barthès et al., 1997). Son plan quasi complet a pu être relevé grâce aux photographies aériennes [ill. 5]. Il s'agit d'un fossé arqué qui s'appuie sur le rebord d'une terrasse alluviale. Il mesure environ $650 \mathrm{~m}$ de long et est formé d'une quinzaine de segments séparés par des interruptions. Les sondages et fouilles ont révélé que ce fossé était doublé par une tranchée de palissade qui le borde à $5 \mathrm{~m}$ vers l'intérieur et qui avait, elle aussi, de multiples petits passages en correspondance avec les interruptions du fossé. Cette enceinte, qui se compose à la fois d'un fossé, d'une levée de terre et d'une palissade, délimitait un espace ovalaire d'environ 12 ha à l'intérieur duquel ont été observées quelques structures ponctuelles. Les fouilles ont révélé la présence de fours à pierres chauffées, de silos réutilisés en dépotoirs, et des restes d'habitations à trous de poteaux, mais toutes ces structures ne sont pas contemporaines de l'enceinte. Poste Vieille correspond au modèle classique du «causewayed enclosure», un type de site ceinturé qui est attesté à des centaines d'exemplaires en Europe occidentale dont il est le représentant le plus méridional. Plusieurs sites du Toulousain présentent des caractéristiques semblables et sont tous attribuables au Chasséen garonnais (42002500 avant notre ère). Bien que leurs plans soient 
3. Lugo di Romagna,

Ravenna, Émilie-Romagne

(Italie). Ce site découvert

dans une carrière d'argile

était enfoui à $14 \mathrm{~m}$ de

profondeur. Il a

probablement été

incendié avant d'être

recouvert par des limons

d'inondation, ce qui a

assuré son excellent état

de conservation.

4. Saint-Michel-du-Touch,

Toulouse, Haute-Garonne.

Les palissades de ce site

furent les premières

identifiées dans le

Chasséen méridional. Les

datations radiométriques

ont révélé que ces

structures limitatives

avaient constitué les

enceintes les plus

anciennes du site qui sont

datées de la première

moitié du ve millénaire

avant notre ère.
Lugo di Romagna
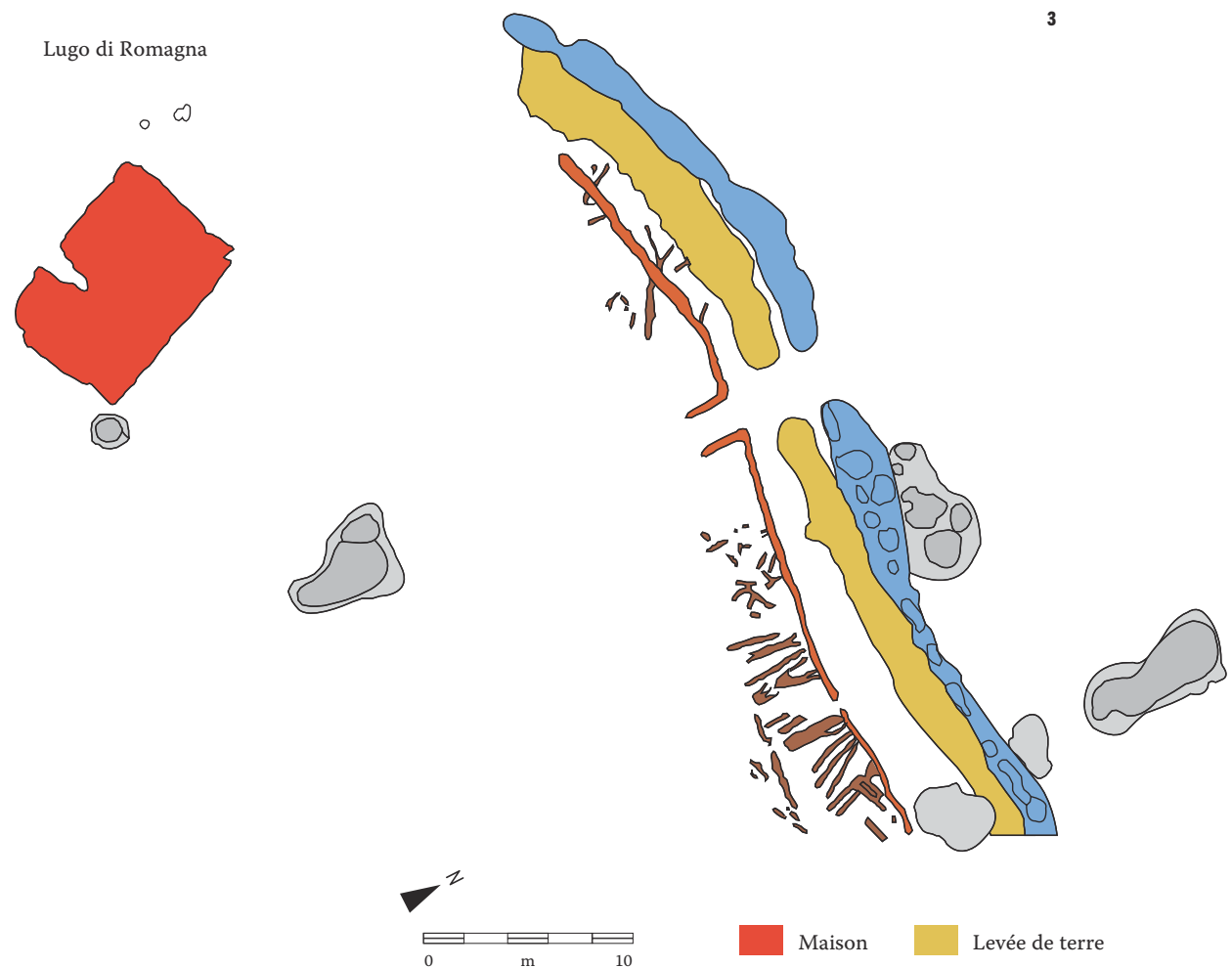

\begin{tabular}{|l|l|}
\hline Maison & Levée de terre \\
\hline Palissade & Fossé \\
\hline Poteaux & Fosses \\
\hline
\end{tabular}

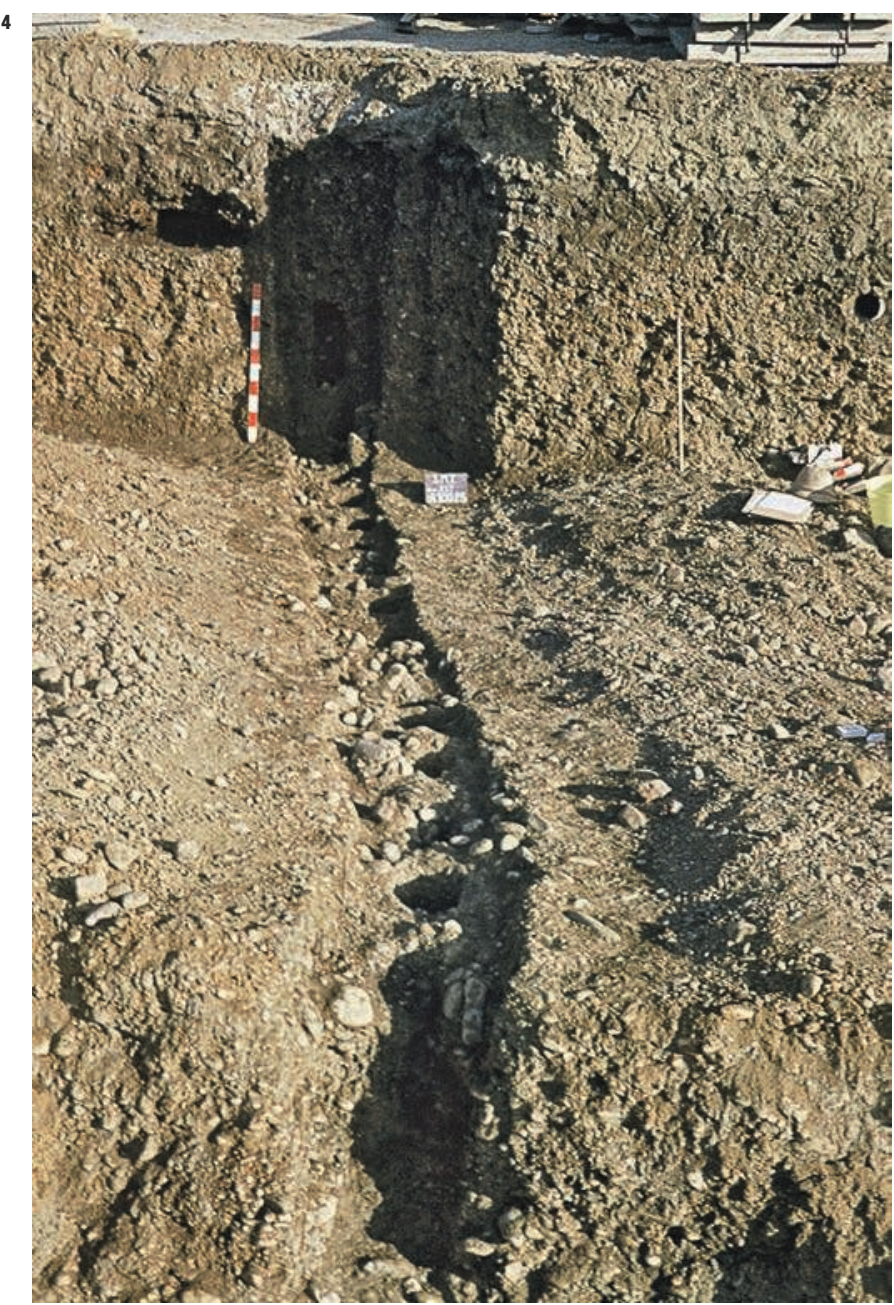


incomplets en raison de leur détection ou fouille au cours de travaux d'urbanisme, on peut affirmer qu'ils comportent eux aussi des fossés doublés de palissades et de levées de terre. Il faut relever à leur propos que les systèmes d'enceinte ont eu tendance à se multiplier sur les mêmes sites au cours de cette période puisqu'on compte au moins cinq systèmes retranchés successifs à Saint-Michel-du-Touch et autant à Villeneuve-Tolosane et Cugnaux, où les abondants vestiges d'occupations s'étendent sur 20 à 40 ha. Il s'agit surtout de fours à galets chauffés avec quelques silos ou fosses qui témoignent d'activités domestiques nombreuses. Les trous de poteaux et vestiges de construction sur poteaux porteurs sont rares et mal documentés et certains auteurs ont considéré que ces enceintes ne seraient pas seulement des villages entièrement habités, mais aussi des pôles de réunion pouvant accueillir périodiquement des groupes dispersés pour des fêtes, des cérémonies ou des échanges. Ces hypothèses peuvent cadrer avec les systèmes les plus grands; elles sont moins pertinentes dans le cas d'autres enceintes d'extension réduite ayant de puissants fossés et une seule interruption comme l'éperon barré d'Auriac à Carcassonne, Aude [ill. 6], dans lequel des sols et des structures d'habitat durables sont bien attestés. Il semble évident que le tronçon de fossé récemment fouillé sur le site de Château-Percin à Seilh en Haute-Garonne ait eu une réelle fonction défensive [ill. 7]. Son comblement est constitué des restes brûlés d'un rempart en bois et en mottes de terre résultant probablement d'un incendie dont la cause pourrait être conflictuelle. Cette enceinte a rapidement été reconstruite juste à côté, comme le suggère le récent programme de datations et études bayésiennes réalisé sur le site dans le cadre du programme TOLT de l'European Research Council (ERC) dirigée par Alasdair Whittle et Alex Bayliss.

Dans la péninsule Ibérique, quelques sites avec des structures périmétrales légères sont attestés dès le Néolithique ancien : c'est le cas notamment du site de La Revilla del Campo à Ambrona près de Soria (Rojo et al., 2008), qui a livré les restes de deux petits enclos faits de tranchées d'implantation de palissades qui délimitent deux espaces circulaires mesurant respectivement $16 \mathrm{~m}$ et $29 \mathrm{~m}$ de diamètre; le plus grand avait une entrée et une double palissade. Ce genre d'enclos est évidemment trop petit pour une limite d'habitat groupé, il cadrerait mieux avec une concession familiale, mais ces structures ont été interprétées comme de possibles monuments cérémoniels au sein d'un habitat ouvert comportant de nombreuses fosses.

\section{Les villages péri-lacustres \\ du Néolithique méditerranéen}

Les habitats péri-lacustres sont limités naturellement par l'eau et la topographie des îles ou presqu'îles sur lesquelles ils sont établis, ce qui permet de circonscrire leur étendue. Parfois, des systèmes de palissades ou de chemins de planches viennent compléter cette délimitation naturelle de l'espace du côté de la terre ferme. Le village lacustre néolithique, tel que son image est véhiculée depuis le $\mathrm{XIX}^{\mathrm{e}}$ siècle, est foncièrement ancré dans le domaine nord alpin où se sont déroulées les nombreuses recherches qui ont abouti à des méthodes de fouille spécifiques combinant l'approche spatiale et chronologique grâce à la stratigraphie, la dendrochronologie et de multiples approches paléoenvironnementales et palethnographiques. Toutefois, l'origine de ce phénomène d'habitat en bord de lac ou en tourbière pourrait être méditerranéenne, si l'on tient compte des trouvailles et des récents programmes de recherches réalisés en contexte humide en Italie, en France et en Espagne. Létablissement lacustre le plus ancien actuellement connu en Méditerranée occidentale est le site de la Marmotta à Anguillara Sabazia (Rome) qui a été découvert par hasard lors de la pose d'une canalisation d'eau au fond du lac volcanique de Bracciano. Situé à $350 \mathrm{~m}$ du rivage actuel et à une profondeur de $8 \mathrm{~m}$, il a fait l'objet d'une fouille subaquatique sur près de $2000 \mathrm{~m}^{2}$, ce qui semble correspondre à un dixième de son extension maximale (Fugazzola Delpino et al., 2004). De la répartition de plusieurs milliers de pieux en chêne conservés dans le limon lacustre ou correspondant à des restes de parois et de toitures effondrées, il a pu être établi que cet établissement comportait des unités d'habitation rectangulaires d'environ 6/8 m. Elles étaient disposées en file, le long d'espaces de circulation orientés parallèlement au rivage du lac. Cet habitat était essentiellement en bois, mais il comportait aussi des empierrements formant des sortes de terrasses et des structures en argile pour les revêtements de murs et des sols. Outre les maisons, il y avait aussi des dépendances telles que des abris à pirogues et des structures de stockage. Les datations dendrochronologiques ont révélé une longue durée d'occupation de 5690 à 5230 avant notre ère. Il est possible que le modèle de tels habitats lacustres ait été diffusé vers le midi de la France au Néolithique ancien, mais on n'a pas trouvé jusqu'à présent de site bien conservé pouvant documenter amplement ces aspects. Dans l'étang de Leucate (Aude), un probable site lacustre du Cardial franco-ibérique a été détruit par les dragues qui ont creusé un port de plaisance. D'autres sites anciennement lacustres ont pu faire l'objet de fouilles, mais l'assèchement historique des anciens lacs ou marais où ils se trouvaient n'a pas permis la conservation des matériaux organiques. C'est le cas notamment du site du Baratin à Courthézon dans le Vaucluse qui se trouve en bordure d'un ancien «palud». Il a livré des sols d'occupation raccordés à des cuvettes remplies de galets chauffés correspondant à des fours, ainsi qu'une grande structure empierrée interprétée initialement comme un sol pavé de cabane ronde. Cette 
vaste enceinte a été

révélé par des

photographies aériennes

obliques qui ont été

redressées. Il correspond

à une enceinte fossoyée et

palissadée qui enclôt une

surface de 12 ha dont une

petite partie a été

décapée et fouillée.
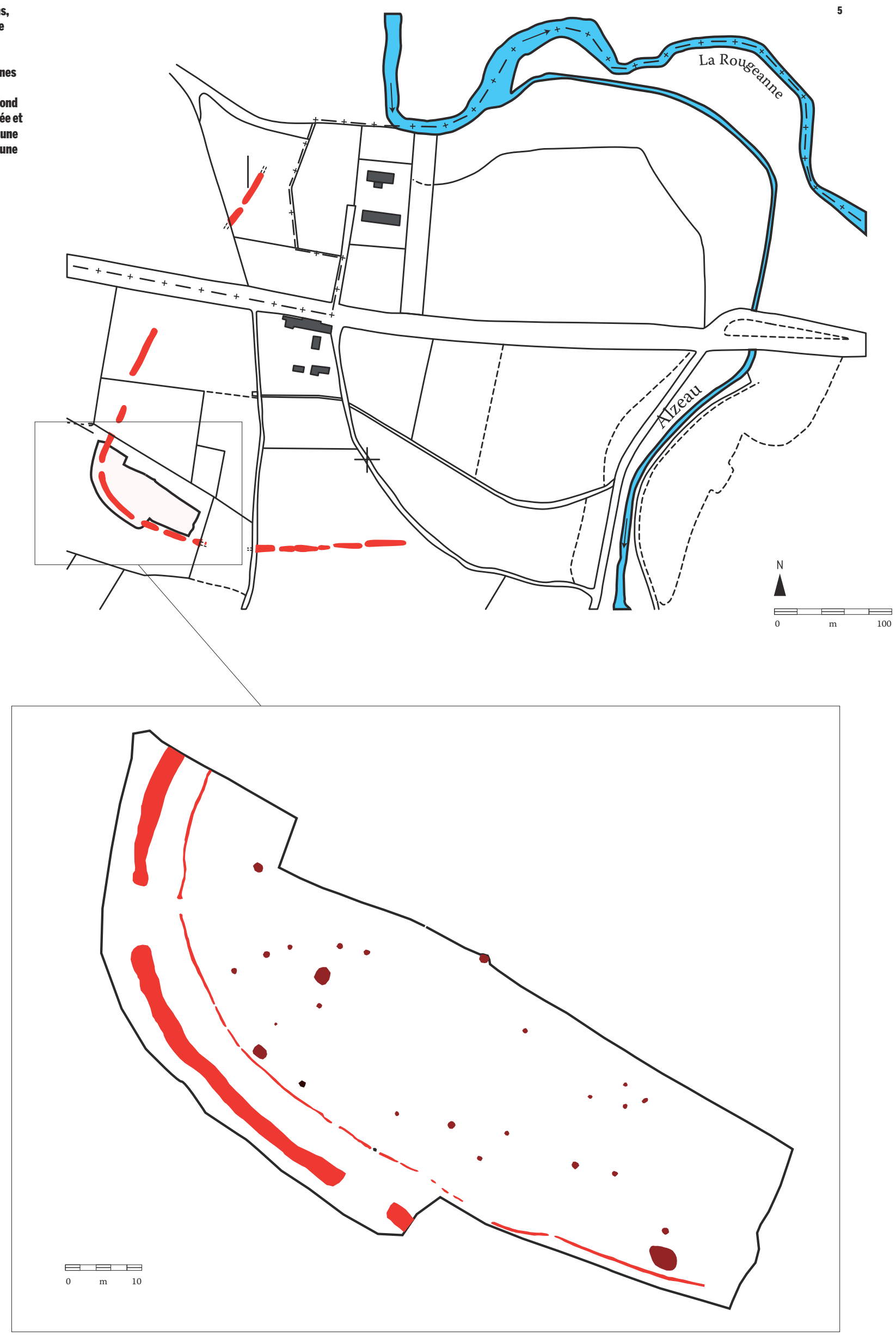
hypothèse a ensuite été remise en cause avec le dégagement de la partie arrière d'un bâtiment à abside qui rappelle la forme de ceux d'Italie du Sud (Binder, Sénepart, 2004). Un autre habitat d'ancienne zone humide a été fouillé lors de la construction du TGV Méditerranée sur le site des Petites Bâties à Lamotte-du-Rhône dans le Vaucluse (Binder et al., 2002). Établi sur une ancienne terrasse du Rhône dans un secteur soumis à des remontées de nappe phréatique, c'est un site qui combine des zones de sol ferme à encroûtement carbonaté et des chenaux limoneux présentant l'aspect d'anciens marais ou de «prairies mouillères ». Ces dépressions ont piégé les témoins de plusieurs occupations du type villageois. La plus ancienne, attribuable au Cardial de style rhodano-provençal, est datée entre 5300 et 5100 avant notre ère. Elle se caractérise par des nappes de vestiges, des trous de poteaux, des structures en cuvette remplies de galets chauffés et des fosses formant une concentration sur plus de 3 ooo $\mathrm{m}^{2}$. La seconde occupation, dégagée en partie seulement, est plus récente et attribuable à l'époque de transition entre le Néolithique ancien et moyen. Datée entre 4900 et 4610 avant notre ère, elle se caractérise par des files de trous de poteaux pouvant correspondre à des palissades, voire à l'implantation d'un chemin de planches. L'habitat est attesté par la présence d'alignements de trous de poteaux, des fosses de stockage et des fours à pierres chauffées.

En Catalogne, un autre village lacustre bien conservé a été révélé à La Draga en bordure du lac de Banyoles (Girona) lors des aménagements liés à la construction d'un parc de loisirs. Les fouilles préventives qui ont suivi ont permis de cerner le site qui occupait une sorte de presqu'île d'environ 8 ooo $\mathrm{m}^{2}$, située en majeure partie sous la berge actuelle du lac et pour un dixième environ sous les eaux du lac actuel (Tarrús, 2008). De nombreuses datations radiométriques ont fixé l'occupation entre 5300 et 5000 avant notre ère, ce qui correspond bien avec l'abondant mobilier retrouvé appartenant au Cardial récent de style catalan. La dendrochronologie, bien qu'en séquence flottante, a permis d'estimer la durée d'occupation à moins d'un siècle. Malgré la présence de centaines de poteaux plantés, les recherches n'ont pas permis jusqu'à présent de bien cerner la forme des habitations en raison du faible développement des analyses dendrologiques qui sont les seules à pouvoir discriminer des ensembles parfaitement synchrones et structurés. Les premiers résultats semblent indiquer qu'il s'agissait de maisons rectangulaires, peut-être organisées en deux alignements parallèles à l'ancien rivage. Dans une autre zone, plus élevée et au sec, se trouvait un espace voué au traitement des céréales et à leur stockage dans de petits greniers.

Faute de trouvailles significatives, on ne connaît pas d'établissement lacustre plus récent dans les zones méridionales. C'est seulement en périphérie des Alpes italiennes que ce genre d'habitat est bien attesté, notamment à Fimon, Molino Casaroto (Vincenza) pour l'étape ancienne de la culture des vases à bouche carrée (Bagolini, Barfield, Broglio, 1973). En Lombardie, d'autres sites lacustres du Néolithique ancien et moyen sont connus, à l'Isolino di Varese ou à la Lagozza di Besnate, mais ils n'ont pas été explorés sur d'assez grandes surfaces pour pouvoir cerner leurs composantes et leur mode d'organisation. Dans le midi de la France, le seul site lacustre alpin bien documenté est le village des Baigneurs à Charavines (Isère). Daté entre 2669 et 2592 avant notre ère, il comportait une délimitation sous forme de palissade qui enserrant un petit habitat groupé d'environ o,2 ha qui n'a jamais comporté plus de quatre à cinq maisonnées (Bocquet, Houot 2012). Sur ce site, la multiplication des analyses xylologiques et des datations dendrochronologiques a permis de discriminer les diverses constructions en bois et de bien préciser les étapes d'édification et de reconstruction des maisons. Il en ressort que l'occupation n'a pas été continue, mais qu'elle est marquée par deux épisodes d'une vingtaine d'années interrompus par une période d'abandon d'une quarantaine d'années. Ce modèle d'occupation discontinue, en gros de la durée d'une génération et séparée par des périodes d'abandon, est attesté dans de nombreux autres sites lacustres et correspond à une économie basée sur les cycles d'abattage et de régénération de la forêt.

\section{Des formes d'organisation différentes du Néolithique moyen au Néolithique final}

Lors du développement du Néolithique en Méditerranée occidentale, plusieurs formes d'habitat sont perceptibles. Elles correspondent sans doute à des entités sociales ou économiques distinctes : des installations troglodytiques pour des groupes spécialisés dans la chasse ou l'élevage, des fermes ou concessions pour des exploitations agricoles familiales et des villages pour des communautés que l'on suppose plurifamiliales. Pour chacune de ces formes, la question de la durée d'occupation et de la composition des groupes humains concernés se pose et ne peut réellement être appréhendée que dans des cas très rares de sites lacustres où l'on peut dénombrer les unités domestiques et leur durée d'utilisation. D'autres questions se posent qui ne peuvent pas vraiment être résolues : elles concernent les liens qui pouvaient exister entre ces diverses formes d'habitat et leur caractère complémentaire. Au Néolithique ancien, dans les régions nouvellement investies par les colons néolithiques, on peut supposer que des installations villageoises stables servaient de bases pour des sites satellites saisonniers et/ou spécialisés. C'est ce modèle qui a été formulé à propos de l'abri Jean-Cros dans les Corbières (Guilaine et al., 1979) ou de la grotte Lombard à Saint-Vallier-de-Thiey (AlpesMaritimes; Binder, 1991). Il est établi que ces grandes catégories de sites ont duré tout au long

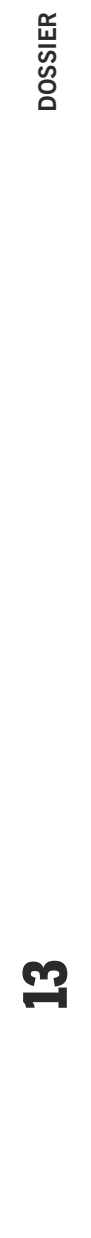


6.Auriac, Carcassonne, Aude. L'éperon d'Auriac, partiellement fouillé, est un habitat du Chasséen classique qui était

protégé par un fossé d'environ $150 \mathrm{~m}$ de long avec une seule

interruption centrale.Ce

fossé de $5 \mathrm{~m}$ de large pour $2 \mathrm{~m}$ de profondeur était doublé par une levée de

terre interne dont

l'existence est déduite du remplissage

dissymétrique du

comblement.

7. Château-Percin, Seilh, Haute-Garonne. Cette enceinte est le témoin exceptionnel d'un

incendie massif ayant entraîné la destruction totale d'un puissant rempart en bois et en terre dont les vestiges en terre, préservés par la

cuisson, se sont effondrés dans le fossé. Ces témoins ont permis de préciser le caractère monumental de cette architecture collective ainsi que les techniques utilisées pour $\checkmark$ la réaliser.
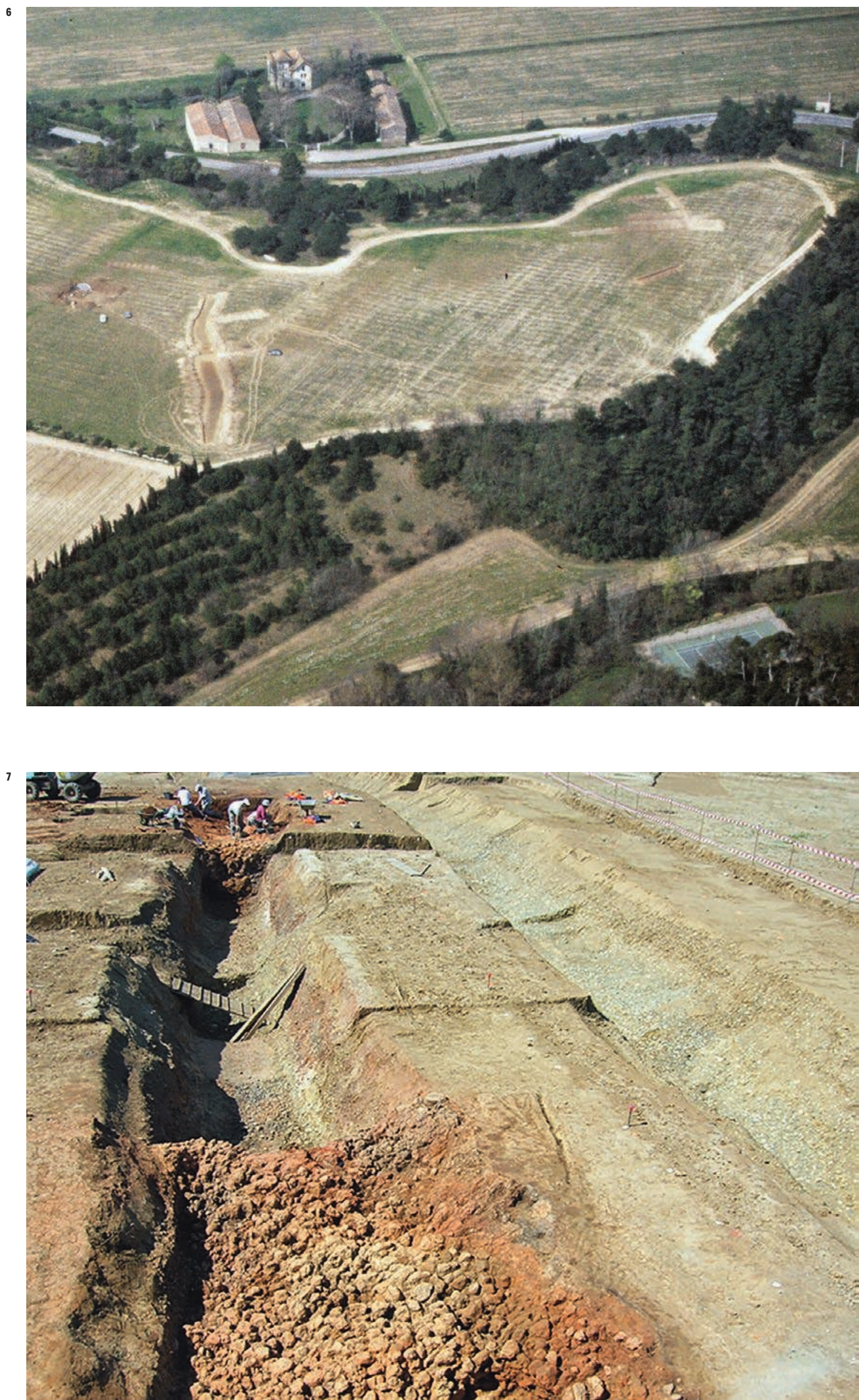

niveau d'organisation supplémentaire de rang proto-urbain. Pendant toute cette durée, c'est le village qui traduit le stade le plus complexe de l'organisation sociale, celui qui révèle des formes de solidarité communautaire pour des réalisations de grande ampleur, comme les enceintes qui constituent les pôles fixes d'une société encore mobile dans des territoires exploités extensivement par des procédés itinérants ou cycliques. Dans de tels contextes, des concurrences et même des conflits ont pu exister pour le contrôle des terroirs et l'on peut s'interroger sur les disparités régionales qui existent entre des territoires à peuplement dispersé, comme ceux de la zone méditerranéenne, et des territoires à peuplement plus concentré dans de grands sites ouverts comme ceux du Chasséen rhodanien et d'autres à sites fortifiés comme ceux de l'Aude ou de la vallée de la Garonne. Il est frappant de constater que les grands sites villageois, fortifiés ou non, se rapportent pour la plupart au Néolithique moyen, tandis qu'ils sont plus rares au Néolithique final. On ne doit pas envisager une baisse démographique entre ces deux périodes puisque, hormis le troisième quart du IV ${ }^{\mathrm{e}}$ millénaire, qui semble avoir été une période de crise écologique et démographique, le nombre de sites occupés au Néolithique final est partout beaucoup plus élevé, atteignant une sorte de paroxysme au Néolithique final 3 (entre 2800-2400 avant notre ère). Ces disparités ne traduisent-elles pas des formes différentes d'organisation sociale entre les deux périodes?

En correspondance avec ces changements, on peut faire appel aux données du registre funéraire qui opposent d'un côté les grands sites chasséens, avec des pratiques funéraires privilégiant les sépultures individuelles, et les petits sites disséminés du Néolithique final chalcolithique qui sont associés à des monuments funéraires collectifs marquant probablement des formes d'appropriation de terroir non par des communautés, mais plutôt par des clans ou lignages.

\section{Références bibliographiques}

Ammerman A., Cavalli-Sforza L., 1984, The Neolithic Transition and the Genetics of Populations in Europe, Princeton, Princeton University Press.

Bagolini B., BARfield L., Broglio A., 1973, «Noticie preliminari delle ricerche sull'insediamento neolitico di Fimon-Molino Casarotto (Vicenza) (1969-1972)», Rivista di Scienze Preistoriche, XXVIII (1), p. 161-215.

Bernabeu Auban J., Orozco Kholer T., Diez Castillo A., Gomez Puche M. Molina Hernandez F.-J., 2003, « Mas d'Is (Penàguila, Alicante). Aldeas y recintos monumentales del Neolitico initial en el valle del Serpis », Trabajos de prehistoria, 6o, 2, 2003, p. 39-59.

BINDER D. (DIR.), 1991, Une économie de chasse au Néolithique ancien. La grotte Lombard à SaintVallier-de-Thiey (Alpes-Maritimes), Paris, éd. du CNRS, $239 \mathrm{p}$.

Binder D., Jallot L., Tiebault S., 2002, «Les occupations néolithiques des Petites Bâties, Lamotte-du-Rhône, Vaucluse », in Archéologie du TGV Méditerranée. Fiches de synthèse, Lattes, Association pour la recherche archéologique en Languedoc oriental (coll. Monographies d'archéologie méditerranéenne, 8-10), t. 1 , p. 103-122.

BINDER D., SÉNÉPART I., 2004, «Derniers chasseurs et premiers paysans de Vaucluse. Mésolithique et Néolithique ancien, 700o-4700 av. J.-C.», in Buisson-Catil J., Guilcher A., Hussy C., Olive M., PAgni M., Vaucluse préhistorique, Le Pontet, A. Barthélemy, p. 127-162.

BocQuet A., Houot A. 2012, Les oubliés du lac de Paladru, ils vivaient depuis 5000 ans $\grave{a}$ Charavines en Dauphiné, Montmélian, La Fontaine de Siloé, $186 \mathrm{p}$.

Bradford J. S., Williams Hunt P.R., 1946, "Siticulosa Apulia », Antiquity, XX, p. 146.

Degaspari N., Ferrari A., Steffe J., 1999, «L'Insediamento neolitico di Lugo di Romagna» in Pessina A. E Muscio G., Settemila anni fa.. il prime pane, ambienti e cultura delle società neolitiche, Udine, Museo friulano di storia naturale, p. 117-124.

Fugazzola Delpino M. A., Pessina A., Tine V. 2004, Civilità dell'argilla. Le prime comunità del neolitico. Catalogo della mostra al museo Pigorini, Roma, Imago Media, $170 \mathrm{p}$.

GANDELIN M., 2011, Les Enceintes chasséennes de Villeneuve-Tolosane et de Cugnaux dans leur contexte du Néolithique moyen européen, Toulouse, Archives d'écologie préhistorique, $506 \mathrm{p}$.

Guilaine J., Barthes P. et al., 1997, La Poste-Vieille, Carcassonne, Centre d'anthropologie des sociétés rurales de Toulouse, Archéologie en Terre d'Aude, $252 \mathrm{p}$.

Guilaine J., Gasco J., Vaquer J., BARbAZA M. (DIR.), 1979, L'Abri Jean-Cros, Toulouse, Centre d'anthropologie des sociétés rurales de Toulouse, $457 \mathrm{p}$.

Rojo Guerra M., Garrido Pena R., Garcia Martinez de Lagran I., Kunst M. 2008, Los Recintos del poblado del Neolítico antiguo de la Revilla del Campo (Ambrona, Soria), Actas del IV Congresso del Neolítico peninsular, Alicante, éd. du Museo de Arqueologia de Alicante, t. 1, p. 252-258.

TARRús J., 2008, «La Draga (Banyoles, Catalonia), an Early Neolithic Lakeside Village in Mediterranean Europe ", Catalan Historical Rewiew, 1, p. 17-33.

Tiné S., 1983, Passo di Corvo e la civiltà neolitica del Tavoliere, Genova, SAGEP, 202 p.

VAlera A. C., 2013, "Cronologia dos recintos de fossos da Pré-historia Recente em território português ", in Arqueologia em Portugal, Lisboa, Associação des Arqueólogos Portugueses, p. 335-343. 